\title{
Sexual risk behaviour trajectories among MSM at risk for HIV in Amsterdam, the Netherlands
}

\author{
Maartje Basten $^{a, b}$, Janneke Cornelia Maria Heijne ${ }^{a}$, \\ Ronald Geskus $^{\mathrm{b}, c, d, e}$, Chantal Den Daas ${ }^{\mathrm{a}, \mathrm{f}}$, Mirjam Kretzschmar ${ }^{\mathrm{a}, \mathrm{g}}$ \\ and Amy Matser
}

\begin{abstract}
Objective: Sexual risk behaviour changes during a person's life course. Insights in sexual risk behaviour trajectories of MSM may provide starting points for the timing of HIV prevention methods. We aimed to study longitudinal trajectories of sexual risk behaviour predictive of HIV acquisition from sexual debut onwards.

Design: A longitudinal study among 815 HIV-negative participants of the Amsterdam Cohort Studies (ACS) who completed extensive questionnaires about their sexual behaviour every 6 months between 2007 and 2017.

Methods: A comprehensive behavioural risk score predictive of HIV seroconversion was developed. On the basis of this risk score, linear trajectories of sexual risk behaviour and MSM group membership were estimated using latent class growth mixture modelling. Associations between longitudinal trajectories and demographic and psychosocial factors were examined.

Results: Three trajectories of sexual risk behaviour were identified, which were labelled Low risk (90.3\% of the sample), Falling high risk (6.5\%) and Rising high risk (3.3\%). MSM following the Falling high risk (20.5\%) and Rising high risk $(25.0 \%)$ trajectories were more likely to acquire HIV during follow-up. The Falling high risk trajectory was associated with younger age at sexual debut, fewer steady partnerships and high percentages of substance use. The Rising high-risk trajectory was associated with increasing percentages of substance use over time.
\end{abstract}

Conclusion: MSM follow different trajectories of changing sexual risk behaviour over time. Early identification of MSM following a trajectory of falling or rising high-risk behaviour and adequate timing of individual-based preventive interventions may reduce HIV transmission. Copyright @ 2018 Wolters Kluwer Health, Inc. All rights reserved.

AIDS 2018, 32:1185-1192

Keywords: follow-up studies, HIV infections, MSM, risk-taking, sexual behaviour, sexual partners

\section{Introduction}

Among MSM, sexual behaviour has been influenced by important population events: after the HIV outbreak in the early 1980 s, a reduction in sexual risk behaviour was observed [1-3], and following the availability of combination antiretroviral therapy in 1996, risk behaviour increased again [4,5]. Within these population trends, sexual risk behaviour also fluctuates during someone's life course. At the individual level, sexual risk

\footnotetext{
${ }^{\mathrm{a}}$ Center for Infectious Diseases Control, National Institute for Public Health and the Environment (RIVM), Bilthoven, ${ }^{\mathrm{b}}$ Department of Infectious Diseases, Research and Prevention, Public Health Service Amsterdam, ${ }^{\mathrm{C}}$ Department of Clinical Epidemiology, Biostatistics and Bioinformatics, Academic Medical Center, Amsterdam, The Netherlands, dNuffield Department of Medicine, University of Oxford, Oxford, UK, ${ }^{\mathrm{e}}$ Oxford University Clinical Research Unit, Wellcome Trust Major Overseas Programme, Ho Chi Minh City, Viet Nam, 'Department of Interdisciplinary Social Science, Faculty of Social and Behavioural Sciences, Utrecht University, and ${ }^{\mathrm{g} J u l i u s}$ Center for Health Sciences and Primary Care, University Medical Center Utrecht, Utrecht, The Netherlands. Correspondence to Amy Matser, PhD, Department of Infectious Diseases, Research and Prevention, Public Health Service of Amsterdam, The Netherlands, Postbox 2200, 1000 CE Amsterdam, The Netherlands.
}

Tel: +31020 5555229; e-mail: amatser@ggd.amsterdam.nl

Received: 14 September 2017; revised: 28 February 2018; accepted: 12 March 2018. 
behaviour might change due to life events, such as starting or ending a steady partnership, mental health problems and substance use [6]. Currently, detailed information on change in sexual risk behaviour over lifetime is limited.

Although each individual MSM has his own trajectory of sexual risk behaviour throughout life, 'typical' patterns of sexual risk behaviour can be identified at the population level [7,8]. Lim et al. [7] examined trajectories of numbers of male sexual partners among older HIV-negative and positive MSM aged 50-60 years. Pines et al. [8] studied trajectories of sexual risk behaviour, represented by a predefined HIV risk score, over calendar time from 2003 to 2011 in HIV-negative MSM. These studies, in which latent class trajectory models were used, showed that distinct longitudinal trajectories of low, moderate and high risk can be identified and that MSM following these trajectories differ from each other. These studies provided important insights into the development of sexual risk behaviour over time. Additional evidence of the existence and development of these sexual risk trajectories during the life course is needed to develop prevention strategies that can inform timing of individual-based interventions, such as prescription of pre-exposure prophylaxis for HIV (PrEP). Tailoring interventions to periods of high-risk behaviour during the life course may improve their effectiveness and save costs.

To our knowledge, we are the first to study longitudinal trajectories of sexual behaviour of HIV-negative MSM during the life course, not over calendar time, using a sexual risk score that is predictive of HIV acquisition. We used longitudinal data from the Amsterdam Cohort Studies on HIV and AIDS (ACS) collected between 2007 and 2017. A comprehensive behavioural risk score that predicts HIV seroconversion in MSM was developed and used in latent class growth mixture modelling (GMM) to obtain sexual risk behaviour trajectories. Men following specific trajectories were then characterized with respect to demographic and psychosocial factors.

\section{Materials and methods}

\section{Study population and procedure}

The ACS among MSM started in 1984 in Amsterdam, the Netherlands, and is an open, ongoing prospective cohort study to investigate the epidemiology, psychosocial determinants, course of infection and pathogenesis of HIV [3]. Men are eligible for participation if they live in or around Amsterdam and had at least one male sexual partner in the preceding 6 months (see also Jansen et al. [4]. Recruitment is done by 'convenience sampling' (e.g. brochures at the STI clinic, advertisements in the gay scene) and 'chain referral sampling' (participants recruited by other participants). Participants visit the Public Health Service of Amsterdam every 6 months to complete a self- administered questionnaire about their sexual behaviour in the preceding 6 months. At each visit, MSM are tested for HIV and other sexually transmitted infections. To test for HIV antibodies, two commercially available ELISAs are used (AxSYM; Abbott Laboratories, North Chicago, Illinois, USA and Vironostika; Organon Teknika, Boxtel, the Netherlands). HIV seroconversion is confirmed by western blot analysis (Genelabs Diagnostics, Singapore). The ACS was approved by the Medical Ethics Committee of the Academic Medical Center, University of Amsterdam, Amsterdam, the Netherlands (MEC 07/182).

The present study comprised MSM who visited the ACS between May 2007 and January 2017. Men were included in the present study if they were HIV negative at the start of the study period in 2007 or when they entered the ACS at a later time point. MSM who seroconverted during the study period were censored after their first HIV-positive visit.

\section{Sexual risk behaviour}

We studied sexual risk behaviour with casual partners, assuming that this would be more discriminatory than behaviour with steady partners. At every visit, detailed questions were asked about anal intercourse with casual partners in the preceding 6 months. For the development of a sexual behaviour risk score, we used numbers of anal intercourse partners, condom use during anal intercourse (reported on a 5-point scale from 1 'always used a condom' to 5 'never used a condom') and number of condomless anal intercourse partners with an unknown or positive HIV status. All questions were asked separately for insertive and receptive anal intercourse and for three types of casual partners: one-night stand ('someone you have met by chance and had sex with only once'), multiple-time casual partner ('someone you have met by chance on several occasions and had sex with on these occasions') and sex buddy ('someone you intentionally contact on a regular basis to have sex with') [9]. We also included information on whether the participant engaged in anal intercourse during group sex (yes/no).

\section{Demographic and psychosocial factors}

Demographic and psychosocial factors were taken into account to characterize longitudinal trajectories: migration background, educational level, age at first visit in the study window, age at sexual debut with a man, having a steady partnership and substance use. Migration background was based on country of birth of each parent and categorized into Dutch and non-Dutch. Educational level was assessed at intake and was considered 'high' with completion of higher vocational education or university, and 'middle and low' with completion of secondary vocational education, high school, basic vocational education or primary school. Steady partnerships and substance use in the preceding 6 months were assessed at each visit. Substance use was split into four binary variables (yes/no): alcohol use, recreational drug use 
(including cocaine, 3,4-methylenedioxymethamphetamine (XTC), ketamine, $\boldsymbol{\gamma}$-hydroxybutyric acid (GHB), $\gamma$-butyrolactone (GBL), amphetamine, mushrooms, methamphetamine and mephedrone), use of poppers during sex and use of erectile dysfunction drugs during sex. Percentages of missing data on demographic and psychosocial factors were below $3 \%$, except for migration background, where $8.2 \%$ of data were missing.

\section{Statistical analyses}

To study longitudinal trajectories of sexual risk behaviour, we first developed a sexual behaviour risk score that is predictive of HIV seroconversion, using Poisson regression analysis in SPSS (IBM SPSS Statistics for Windows, version 21.0; IBM Corp., Armonk, New York, USA). The date of seroconversion was defined as the midpoint between the last HIV-negative and first HIV-positive visit. We performed univariable and multivariable Poisson regression analyses including sexual behaviour variables that were known to be associated with HIV seroconversion. Given the limited number of seroconversions during follow-up, we could include a limited number $(\leq 6)$ of variables. Therefore, we aggregated sexual behavioural information into combined variables and included the variables that were most predictive of HIV seroconversion into our final model (a detailed description of the variables is available in Table 1 of Supplemental Digital Content 1, http://links.lww.com/QAD/B247). Visits with an HIV test, but missing behavioural information, were included and behavioural data from the previous visit within the past year was used. Regression coefficients from the multivariable model were used to calculate a behavioural risk score. Performance of the risk score in predicting HIV seroconversion was examined by calculating the area under the curve of the receiver operating characteristic, which is a plot of the true positive risk and the false positive risk, using the validate function of the rms package in R [10]. We adjusted for optimism using 100 bootstrap draws.

Trajectories of sexual risk behaviour over time were estimated using latent class GMM [11] in Mplus version 7.4 (Muthen \& Muthen 1998-2015). GMM estimates latent class growth curve patterns, allowing for withinclass variation in individual growth trajectories. Longitudinal data were restructured as a function of years since sexual debut. Participants with missing information on sexual debut and visits with missing behavioural data were excluded from analyses. We obtained maximum likelihood parameter estimates with standard errors corrected for nonnormality using a sandwich estimator [12]. We estimated models with one to five latent class trajectories with an intercept, slope and quadratic slope. Interindividual variation was allowed by using random intercept and slopes. To determine the number of latent class trajectories that best fitted the data, we used four criteria [13]: Bayesian information criterion (BIC) with lower values indicating better fit, entropy with values between 0.80 and 1 indicating good classification [14], classes should include a substantial percentage of participants $(>2 \%)$ and an additional trajectory should differ considerably in intercept or slope from other trajectories. From the final model, we retrieved for each participant the most likely latent class trajectory membership.

Finally, we characterized MSM following each trajectory by demographic and psychosocial factors and examined whether the identified trajectories were supported by differences on these factors. Differences between groups were examined by performing analysis of variance (ANOVA) for continuous variables and Chi-squared tests for dichotomous variables. MSM with missing information on demographic or psychosocial factors were excluded. In addition, for each group, we studied change in steady partnership and substance use over time, by comparing the first and last visit within the study window using exact binomial McNemar's tests. These analyses were performed in MSM with at least two visits.

\section{Results}

\section{Sample characteristics}

The total sample consisted of 815 MSM with 9075 visits, with a median number of 12 visits (interquartile range: $5-$ 18) and an average follow-up length of 5.34 years (sd 3.26; see Table 1) per individual, and an average gap length of 197 days (sd 65) between visits. The majority of the MSM had a Dutch background (75.7\%) and was highly educated $(75.4 \%)$. Mean age at first visit was 35.6 years (sd 9.6). During follow-up, 52 men seroconverted.

\section{Behavioural risk score for HIV seroconversion}

The behavioural risk score was developed using data from 815 MSM during 8625 visits, excluding intake visits $(n=310)$ and visits with time since last visit more than 1 year $(n=140)$ from analysis. Final predictors included in the risk score were total number of receptive anal intercourse partners, three combined variables of the number of receptive anal intercourse partners and condom use per partner type (i.e. one-night stands, multiple-time casual partners and sex buddies), total number of condomless insertive anal intercourse partners with unknown or positive HIV status and anal intercourse during group sex (see Table 2 of Supplemental Digital Content 1, http://links.lww.com/QAD/B247, which shows results from univariable and multivariable Poisson models). The estimated risk score varied between 0 (no risk) and 4.87 and the mean risk score was 0.53 (SD 0.74). The maximum risk score, 4.87, corresponds to a probability to acquire HIV within 1 year of 0.56 . The area under the curve of the receiver operating characteristic was 0.77 , indicating that the risk score predicted HIV acquisition fairly accurately. 
Table 1. Sample characteristics.

\begin{tabular}{|c|c|}
\hline & $N=815$ \\
\hline Number of visits, median (IQR) & $12(5-18)$ \\
\hline Average follow-up length in years (SD) & $5.34(3.26)$ \\
\hline Migration background, $n(\%)$ Dutch & $566(75.7)$ \\
\hline Education, $n(\%)$ high & $603(75.4)$ \\
\hline Age $^{\mathrm{a}}$, mean $(\mathrm{SD})$ & $35.6(9.6)$ \\
\hline Age sexual debut, mean (SD) & $18.2(4.2)$ \\
\hline HIV seroconversions during follow-up, $n(\%)$ & $52(6.4)$ \\
\hline \multicolumn{2}{|c|}{ Partnership and sexual behavior in the past 6 months $^{\mathrm{a}}$} \\
\hline Steady partnership, $n(\%)$ & $507(62.2)$ \\
\hline $\begin{array}{l}\text { Number of casual insertive } \mathrm{Al} \text { partners, } \\
\text { mean }(\mathrm{SD}) / \text { median }(\mathrm{IQR})\end{array}$ & $3.27(7.48) / 1(0-3$ \\
\hline $\begin{array}{l}\text { Number of casual receptive Al partners, } \\
\text { mean (SD)/median (IQR) }\end{array}$ & $2.98(7.80) / 0(0-3$ \\
\hline Condomless $\mathrm{Al}^{\mathrm{b}}$ with casual partner, $n(\%)$ & $181(22.9)$ \\
\hline $\mathrm{Al}^{\mathrm{b}}$ during group sex, $n(\%)$ & $211(26.2)$ \\
\hline \multicolumn{2}{|l|}{ Substance use in the past 6 months $\mathrm{s}^{\mathrm{a}}$} \\
\hline Alcohol, $n(\%)$ & $666(82.7)$ \\
\hline Recreational drugs ${ }^{\mathrm{c}}, n(\%)$ & $314(39.3)$ \\
\hline Poppers, $n(\%)$ & $306(38.3)$ \\
\hline Erectile dysfunction drugs, $n(\%)$ & $170(21.3)$ \\
\hline
\end{tabular}

$\mathrm{Al}$, anal intercourse; IQR, interquartile range.

a Reported on the first visit within the study period 2007-2017.

${ }^{b}$ Insertive or receptive anal intercourse.

'Including cocaine, XTC, ketamine, GHB, GBL, amfetamine, mushrooms, methamfetamine and mephedrone.

\section{Longitudinal trajectories of sexual risk behaviour} GMM models were estimated using data from 803 MSM during 8198 visits. Twelve MSM with missing information on sexual debut ( $n=132$ visits) and 745 visits with missing or incomplete behavioural data were excluded. Models with one to five trajectories were estimated including an intercept and slope for each trajectory (fit statistics are shown in Table 3 of Supplemental Digital
Content 1, http://links.lww.com/QAD/B247). Adding a quadratic slope to the models led to nonconvergence. The best fitting model included three latent class trajectories with significant changes (all $P_{\mathrm{S}}<0.001$ ) in risk behaviour over time (Fig. 1): a Low-risk trajectory of low sexual risk behaviour with a small increase over time (90.3\% of the sample); a Falling high-risk trajectory $(6.5 \%)$ with very high levels of sexual risk behaviour during the first years after sexual debut and a decrease to low risk in 30 years; and a Rising high-risk trajectory (3.3\%) with low levels of sexual risk behaviour in the first years after sexual debut and a rapid increase over time. Average posterior probabilities of group membership indicated good classification quality ( 0.97 for Low risk, 0.83 for Falling high risk and 0.85 for Rising high risk). Mean number of visits to the ACS did not differ across trajectories. Over the course of follow-up, 25\% of MSM in the Rising high-risk group $(n=5)$ acquired HIV. The incidence rate in this group was 44.6 per 1000 personyears (py). The incidence rate in the Falling high-risk group was 40.1 per 1000 py and $20.5 \%(n=9)$ acquired HIV, which was significantly higher than the number of MSM in the Low-risk group who acquired $\operatorname{HIV}(n=35$; $4.7 \%, P<0.001$; Table 2). The incidence rate in the latter group was 8.5 per 1000 py.

\section{Associations between trajectories and demographic and psychosocial factors}

MSM in the three groups did not differ significantly from each other according to migration background, educational level and age (Table 2), but differences were found for age at sexual debut, having a steady partnership and substance use $(P<0.001)$. Regarding MSM in the

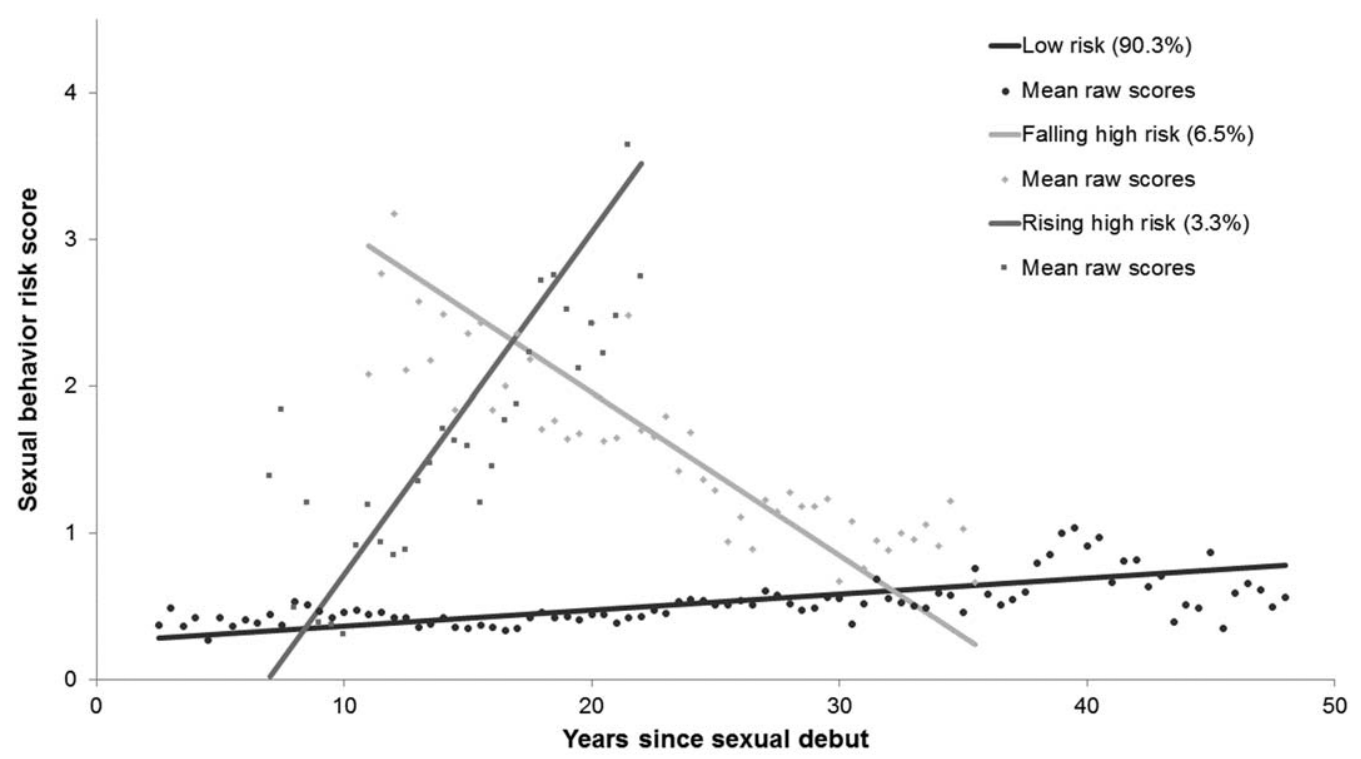

Fig. 1. Estimated longitudinal trajectories of sexual risk behaviour based on latent class growth mixture modelling and mean raw scores within each latent class. $n=803$. Sexual behaviour risk score ranged from 0.00 to 4.87 (mean 0.53, SD: 0.74). Length of plotted trajectories depends on observed values present in each group. 
Table 2. Characteristics of MSM following Low risk, Falling high-risk and Rising high-risk trajectories and differences between groups.

\begin{tabular}{lcccr}
\hline & \multicolumn{3}{c}{ Descriptives } & Differences across groups \\
\cline { 2 - 4 } & $\begin{array}{c}\text { Low risk } \\
n=739\end{array}$ & $\begin{array}{c}\text { Falling high risk } \\
n=44\end{array}$ & $\begin{array}{c}\text { Rising high risk } \\
n=20\end{array}$ & $\begin{array}{c}\text { Overall } \\
P\end{array}$ \\
\hline Ethnicity, \% Dutch & 75.6 & 76.7 & 72.2 & 0.932 \\
Education, \% high & 75.4 & 72.1 & 80.0 & 0.788 \\
HIV seroconversion, \% & 4.7 & 20.5 & 25.0 & $<0.001$ \\
Age, mean (SD) & $35.8(9.7)$ & $34.4(7.4)$ & $31.5(5.4)$ & 0.090 \\
Age sexual debut, mean (SD) & $18.4(4.2)$ & $15.5(3.7)$ & $19.6(3.3)$ & $<0.001$ \\
\hline
\end{tabular}

Falling high-risk group, we found that they were younger at their sexual debut than MSM in the Low-risk group (mean 15.5 vs. 18.4 years, $P<0.001$ ). At their first visit, they were less likely to have a steady partner than MSM in the Low-risk group ( 39.5 vs. $62.9 \%, P=0.002)$, while at the last visit, no difference in steady partnership between groups was found (Table 3). We found that MSM in the Falling high-risk group more often used recreational drugs, poppers and erectile dysfunction drugs than MSM in the Low-risk group at both first visit and last visit $(P=0.010$ to $<0.001)$. Furthermore, among Low-risk MSM, the use of alcohol $(P=0.004)$ and erectile dysfunction drugs $(P<0.001)$ significantly increased between the first and last visit. MSM in the Falling high-risk group showed a decrease in poppers use over time $(75.0 \%$ at first visit vs. $58.1 \%$ at last visit, $P=0.039)$ and recreational drug use and the use of erectile dysfunction drugs increased among Rising high-risk $\operatorname{MSM}(P=0.031$ and $P=0.039$, respectively).

Regarding MSM in the Rising high-risk group, we found that they were more likely to have a steady partnership at their first visit than MSM in the low-risk group (90.0 vs. $62.9 \%, P=0.013$ ), while at the last visit, no difference was present. In the Rising high-risk group, increasing percentages of MSM using recreational drugs $(57.9 \%$ at first visit vs. $95.0 \%$ at last visit $P=0.016)$ and erectile dysfunction drugs $(35.0 \%$ at first visit vs. $70.0 \%$ at last visit, $P=0.039)$ were found over time. At the last visit, MSM in the Rising high-risk group were more likely to use recreational drugs, poppers and erectile dysfunction drugs than MSM in the Low-risk group $(P<0.001)$.

\section{Discussion}

Using a sexual behaviour risk score that is predictive of HIV seroconversion, we found three typical trajectories of sexual risk behaviour during the life course of MSM: Low risk, Falling high risk and Rising high risk. MSM following these different patterns differed not only in their sexual risk behaviour and their risk to acquire HIV but also in age at sexual debut, substance use and being in a steady partnership.

Main strengths of the current study are the large sample of HIV-negative MSM, the wide age range of the study sample (18-70 years), long duration of follow-up in a relatively stable period in which HIV-related events that have a large impact on sexual behaviour were absent and the availability of detailed sexual risk behaviour information. There are also limitations. First, the identified trajectories to describe self-reported sexual risk behaviour are linear, which is a simplification of the more complex trajectories in the real world wherein

Table 3. Differences in steady partnerships and substance use between and within MSM following Low-risk, Falling high-risk and Rising high-risk trajectories.

\begin{tabular}{|c|c|c|c|c|c|c|c|c|}
\hline & \multirow[b]{2}{*}{ Visit ${ }^{\mathrm{a}}$} & \multicolumn{3}{|c|}{ Descriptives } & \multirow{2}{*}{$\begin{array}{c}\text { Difference across groups } \\
\text { Overall } \\
P\end{array}$} & \multicolumn{3}{|c|}{ Difference between first and last visit } \\
\hline & & $\begin{array}{c}\text { Low risk } \\
n=713 \\
\% \text { yes }\end{array}$ & $\begin{array}{c}\text { Falling high } \\
\text { risk } n=44 \\
\% \text { yes }\end{array}$ & $\begin{array}{c}\text { Rising high } \\
\text { risk } n=20 \\
\% \text { yes }\end{array}$ & & $\begin{array}{l}\text { Low } \\
\text { risk } \\
P\end{array}$ & $\begin{array}{c}\text { Falling } \\
\text { high risk } \\
P\end{array}$ & $\begin{array}{c}\text { Rising } \\
\text { high risk } \\
\quad P\end{array}$ \\
\hline \multirow[t]{2}{*}{ Steady partnership } & First & 62.9 & 39.5 & 90.0 & $<0.001$ & & & \\
\hline & Last & 66.0 & 58.1 & 65.0 & 0.570 & 0.109 & 0.092 & 0.125 \\
\hline \multirow[t]{2}{*}{ Alcohol } & First & 83.1 & 79.5 & 90.0 & 0.585 & & & \\
\hline & Last & 87.4 & 76.2 & 85.0 & 0.110 & 0.004 & 0.687 & $-{ }^{\mathrm{b}}$ \\
\hline \multirow[t]{2}{*}{ Recreational drugs } & First & 36.9 & 70.5 & 57.9 & $<0.001$ & & & \\
\hline & Last & 37.1 & 65.1 & 85.0 & $<0.001$ & 1.000 & 0.754 & 0.031 \\
\hline \multirow[t]{2}{*}{ Poppers } & First & 36.4 & 75.0 & 75.0 & $<0.001$ & & & \\
\hline & Last & 38.3 & 58.1 & 90.0 & $<0.001$ & 0.281 & 0.039 & $-{ }^{b}$ \\
\hline \multirow[t]{2}{*}{ Erectile dysfunction drugs } & First & 18.2 & 65.9 & 35.0 & $<0.001$ & & & \\
\hline & Last & 25.5 & 59.5 & 70.0 & $<0.001$ & $<0.001$ & 0.581 & 0.039 \\
\hline
\end{tabular}

${ }^{a}$ Data presented were collected at the first visit within the study period 2007-2017 or at the last visit within the study period $2007-2017$.

${ }^{\mathrm{b}} \mathrm{McNemar}$ 's test could not be performed because number of discordant individuals was too small $(n<6)$. 
periods of higher and lower risk may alternate. The addition of quadratic terms to our model, to allow for nonlinear behavioural patterns during the life course, led to nonconvergence. Possibly, 10 years of follow-up are too short to study nonlinear behavioural changes across the total length of MSM's sexually active life. Also, we had limited data on sexual behaviour during the first years after sexual debut and during the older ages, which resulted in a restricted length of the trajectories. Second, MSM who acquired HIV between sexual debut and inclusion are missed in this analysis, which might have caused an underestimation of MSM with high sexual risk behaviour early in their sexual career and a small bias in estimated group sizes, but not necessarily in the observed behavioural patterns. Third, GMM may result in invalid classes if data are not normally distributed. In the Falling high-risk and Rising high-risk classes, data were rather normally distributed, while the Low-risk class included a preponderance of zero's, that is no risk behaviour with casual partners. We considered this as a valid finding: MSM with low risk have often periods in which they do not engage in any risk behaviour. Fourth, in deciding on the number of latent class trajectories, both parsimony and interpretability of the classes played a role, which may be subjective. We used statistical and substantive criteria in agreement with other studies [13]. The final model contained three classes of different sizes; the majority of MSM were in the Low-risk group and 9\% of MSM belonged to the high-risk groups. Given the low number of individuals in the high-risk groups, the power to detect differences was low. Another limitation involves the overrepresentation of highly educated MSM with a Dutch background, which may limit the generalizability to the entire Dutch MSM population. Also, participants to the ACS study are a motivated group that come for STI and HIV testing every 6 months and may be more aware of their risks than MSM in general. However, behavioural outcomes from the ACS have previously been found to be similar to larger nation-wide monitoring studies, such as the Schorer Monitor [15]. Finally, we did not examine the influence of PrEP use on changes in sexual risk behaviour. In the Netherlands, PrEP use was approved in 2016 and is, due to high costs, only used via informal routes or via participation in the Amsterdam PrEP project, which started only in 2015 [16]. Therefore, it may have had only a little effect on our findings. The influence of PrEP use on change in sexual risk behaviour over time requires attention in future studies.

The trajectories identified in the current study can be interpreted as three general patterns that are currently seen in the MSM population. The trajectories differ from previous studies in which trajectories differed predominantly in severity (low, moderate and high risk) but showed little change in behaviour over time $[7,8]$. Most notably, in our study, a small group of MSM followed a trajectory of rising high-risk behaviour. This group, of whom $25 \%$ acquired HIV, was not previously identified.
Also contrary to others $[7,8]$, we found a slight increase in risk behaviour over time since sexual debut in the Lowrisk group. Time since sexual debut is related to calendar time and the increase in risk behaviour may reflect an ongoing tendency of decreased fear of HIV over calendar time due to effective biomedical interventions $[4,17]$. Of note, even though the HIV incidence rate and risk behaviour with casual partners in the Low-risk group was relatively low, most of the HIV seroconversions occurred in this group. Finally, decreasing sexual risk behaviour was also found by others, although somewhat less pronounced as in our Falling high-risk trajectory $[7,8]$. Several factors may explain the detection of trajectories with changing risk levels over time in the current study and the relatively stable trajectories found by others $[7,8]$. First, the use of a comprehensive continuous behavioural risk score including multiple sexual behaviour characteristics in the current study may have been more sensitive to observe change over time than studying changes in number of sexual partners only [7] or using a dichotomized risk score [8]. Second, in our model, growth factors were random, allowing between-individual differences in intercept and slope within each trajectory, while other studies used latent class growth analysis with growth factors fixed to be the same for each individual within the same trajectory $[11,18]$. Alternative factors that may have played a role are use of different time scales, differences in population characteristics including the large age range in our study and the use of more recent data up to 2017 in the current study.

There was an agreement between the identified trajectories and psychosocial factors that are known to be associated with sexual risk behaviour and HIV acquisition. Compared with Low-risk MSM, MSM in the Falling high-risk group were less likely to have a steady partner at the first visit, while MSM in the Rising high-risk group were more likely to have a steady partner. Possibly, having a steady partner reduces sexual risk behaviour with casual partners, as was also found by others [19-21]. This does not mean, however, that steady partnership is a protective factor for HIV acquisition per se, as a large proportion of HIV transmission among MSM is attributable to steady partners [22,23]. Associations between trajectories and substance use are also in line with previous studies [7,8,24,25]: the Falling high-risk trajectory was associated with high percentages of substance use, with a small decrease in poppers use over time, while for the Rising high-risk group, an increase in MSM using substances was found. Drug use may elicit sexual risk behaviour [24,25], although causal associations remain poorly understood. Finally, MSM in the Falling high-risk group were younger at their sexual debut, which is a known predictor of sexual risk behaviour [26,27]. Agreement between our findings and existing literature supports the validity of the trajectories.

In the current study, we were not able to identify the reasons for or predictors of future changes in risk 
behaviour during the life course. This should be subject of future research, because it may provide starting points for early detection of MSM who will follow a trajectory of high risk. In clinical practice, target groups for interventions are often defined on the basis of their behaviour at the current point in time. Understanding of their sexual risk behaviour trajectories offers the opportunity to tailor individual-based interventions to specific periods in time and identify individuals already before they increase their risk behaviour. This is especially important for interventions such as PrEP, where life-long use is not desirable and might not be feasible, due to high costs, medication overuse and adherence difficulties [28]. Especially for MSM following a Rising high-risk trajectory, early identification can prevent HIV infection.

In conclusion, our study showed that different trajectories of changing sexual risk behaviour can be identified in the MSM population. Differences in demographic and psychosocial characteristics supported the validity of the trajectories. This knowledge might be important to optimize the impact of prevention strategies offered to MSM following different trajectories.

\section{Acknowledgements}

The authors acknowledge all participants of the ACS for their contribution, research nurses Leann Story, Samantha de Graaf and Wendy van der Veldt for data collection and cohort management and Gerben-Rienk Visser and Linda May for data management.

All authors contributed to the study concept. M.B. analysed and interpreted the data, and wrote the draft article. A.M. and J.H. supervised the overall study and contributed to the interpretation of the data. R.G. supervised the analyses and interpretation of the data. C.d.D. and M.K. provided substantial contributions to the interpretation of the data. All authors contributed to subsequent drafts and approved the final version of the article for submission.

This project was funded by the Netherlands Organisation for Health Research and Development ZonMw grant 522002004 .

\section{Conflicts of interest}

The authors declare no conflicts of interest.

\section{References}

1. Winkelstein W, Wiley JA, Padian NS, Samuel M, Shiboski S, Ascher MS, et al. The San Francisco Men's Health Study: continued decline in HIV seroconversion rates among homosexual/bisexual men. Am J Public Health 1988; 78:1472-1474.
2. Winkelstein W, Samuel M, Padian NS, Wiley JA, Lang W, Anderson RE, et al. The San Francisco Men's Health Study: III. Reduction in human immunodeficiency virus transmission among homosexual/bisexual men, 1982-86. Am J Public Health 1987; 77:685-689.

3. van Griensven GJ, de Vroome EM, Goudsmit J, Coutinho RA. Changes in sexual behaviour and the fall in incidence of HIV infection among homosexual men. BMJ 1989; 298:218-221.

4. Jansen IA, Geskus RB, Davidovich U, Jurriaans S, Coutinho RA, Prins $M$, et al. Ongoing HIV-1 transmission among men who have sex with men in Amsterdam: a 25-year prospective cohort study. AIDS 2011; 25:493-501.

5. Rietmeijer CA, Patnaik JL, Judson FN, Douglas JM. Increases in gonorrhea and sexual risk behaviors among men who have sex with men: a 12-year trend analysis at the Denver Metro Health Clinic. Sex Transm Dis 2003; 30:562-567.

6. Huebner DM, Perry NS. Do behavioral scientists really understand HIV-related sexual risk behavior? A systematic review of longitudinal and experimental studies predicting sexual behavior. Arch Sex Behav 2015; 44:1915-1936.

7. Lim SH, Christen CL, Marshal MP, Stall RD, Markovic N, Kim $\mathrm{KH}$, et al. Middle-aged and older men who have sex with men exhibit multiple trajectories with respect to the number of sexual partners. AIDS Behav 2012; 16:590-598.

8. Pines HA, Gorbach PM, Weiss RE, Shoptaw S, Landovitz RJ, Javanbakht M, et al. Sexual risk trajectories among MSM in the United States: implications for preexposure prophylaxis delivery. I Acquir Immune Defic Syndr 2014; 65:579-586.

9. van den Boom W, Stolte I, Sandfort T, Davidovich U. Serosorting and sexual risk behaviour according to different casual partnership types among MSM: the study of one-night stands and sex buddies. AIDS Care 2012; 24:167-173.

10. Harrell FEJ. rms: regression modeling strategies. R package version 5 1-1; 2017. https://cran.r-project.org/web/packages/ $\mathrm{rms} / \mathrm{rms}$.pdf. [Accessed 4 April 2018].

11. Jung T, Wickrama KAS. An introduction to latent class growth analysis and growth mixture modeling. Soc Person Psychol Compass 2008; 2:302-317.

12. Yuan KH, Bentler PM. Three likelihood-based methods for mean and covariance structure analysis with nonnormal missing data. In: Sobel ME, Becker MP, editors. Sociological methodology 2000 Washington, DC: ASA; 2000. pp. 165-200.

13. Collins LM, Lanza ST. Latent class and latent transition analysis. Hoboken, New Jersey: John Wiley \& Sons; 2010.

14. Clark SL, Muthèn B. Relating latent class analysis results to variables not included in the analysis.2009, http://www.statmodel. $\mathrm{com} /$ download/relatinglca.pdf [Accessed 4 April 2018].

15. Van Empelen P, Van Berkel M, Roos E, Zuilhof W. Schorer monitor. Amsterdam, the Netherlands: Schorer; 2011.

16. Hoornenborg E, Achterbergh RCA, Schim van der Loeff MF, Davidovich U, Hogewoning A, de Vries HJC, et al. MSM starting preexposure prophylaxis are at risk of hepatitis $\mathrm{C}$ virus infection. AIDS 2017; 31:1603-1610.

17. Kalichman SC, Price D, Eaton LA, Burnham K, Sullivan M, Finneran $S$, et al. Diminishing perceived threat of AIDS and increasing sexual risks of HIV among men who have sex with men, 1997-2015. Arch Sex Behav 2017; 46: 895-902.

18. Twisk J, Hoekstra T. Classifying developmental trajectories over time should be done with great caution: a comparison between methods. I Clin Epidemiol 2012; 65:1078-1087.

19. Klausner JD, Pollack LM, Wong W, Katz MH. Same-sex domestic partnerships and lower-risk behaviors for STDs, including HIV infection. I Homosex 2006; 51:137-144.

20. Rosenberg ES, Sullivan PS, Dinenno EA, Salazar LF, Sanchez $\mathrm{TH}$. Number of casual male sexual partners and associated factors among men who have sex with men: results from the National HIV Behavioral Surveillance system. BMC Public Health 2011; 11:189.

21. van den Boom W, Davidovich $U$, Heuker J, Lambers F, Prins $M$, Sandfort $T$, et al. Is group sex a higher-risk setting for HIV and other sexually transmitted infections compared with dyadic sex among men who have sex with men? Sex Transm Dis 2016; 43:99-104.

22. Davidovich U, de Wit J, Albrecht N, Geskus R, Stroebe W, Coutinho R. Increase in the share of steady partners as a source of HIV infection: a 17-year study of seroconversion among gay men. AIDS 2001; 15:1303-1308. 
23. Sullivan PS, Salazar L, Buchbinder S, Sanchez TH. Estimating the proportion of HIV transmissions from main sex partners among men who have sex with men in five US cities. AIDS 2009; 23:1153-1162.

24. Colfax G, Vittinghoff E, Husnik MJ, McKirnan D, Buchbinder S, Koblin B, et al. Substance use and sexual risk: a participant- and episode-level analysis among a cohort of men who have sex with men. Am / Epidemiol 2004; 159:1002-1012.

25. Hoenigl M, Chaillon A, Moore DJ, Morris SR, Smith DM, Little SJ. Clear links between starting methamphetamine and increasing sexual risk behavior: a cohort study among men who have sex with men. J Acquir Immune Defic Syndr 2016; 71:551-557.
26. Nelson KM, Gamarel KE, Pantalone DW, Carey MP, Simoni JM. Sexual debut and HIV-related sexual risk-taking by birth cohort among men who have sex with men in the United States. AIDS Behav 2016; 20:2286-2295.

27. Liu Y, Qian HZ, Amico KR, Liu H, Yin L, Ruan Y, et al. Subsequent sexual risks among men who have sex with men may differ by sex of first partner and age at sexual debut: a cross-sectional study in Beijing, China. AIDS Behav 2017; 21:2913-2923.

28. Liu AY, Buchbinder SP. CROI 2017: HIV epidemic trends and advances in prevention. Top Antivir Med 2017; 25: 35-50. 